\title{
Feasibility of the Frequency Planning for LDACS Air-to-Air Communications in the L-band
}

\author{
Miguel A. Bellido-Manganell \\ German Aerospace Center (DLR) \\ Wessling, Germany \\ Miguel.BellidoManganell@dlr.de \\ orcid.org/0000-0002-0668-3477
}

\author{
Michael Schnell \\ German Aerospace Center (DLR) \\ Wessling, Germany \\ Michael.Schnell@dlr.de
}

\begin{abstract}
The 960-1215 MHz frequency range in the L-band is allocated worldwide on a primary basis to the aeronautical radio navigation service (ARNS). At the World Radio Conference 2007, the frequency range $960-1164 \mathrm{MHz}$ within the L-band was additionally allocated to the aeronautical mobile (route) service $(A M(R) S)$ on a co-primary basis to allow future communication systems to share large parts of the $L$-band with the existing radio navigation services. The $L$-band digital aeronautical communications system (LDACS) will operate its air-ground (A/G) and air-to-air (A2A) data links under $A M(R) S$ allocation in the L-band ensuring spectrum sharing without mutual harmful interference. In this paper, we propose a frequency planning methodology for the LDACS A2A data link yielding no harmful interference towards the legacy systems operating in the L-band. We also assess the feasibility of the LDACS A2A frequency planning in the north-east coast of North America, the North Atlantic Corridor, and western Europe. Our results indicate that LDACS A2A can operate in the 960-1164 MHz frequency range without affecting the proper operation of the legacy systems. Whilst the available spectrum is maximized in oceanic airspace and reduced in continental airspace, LDACS A2A can employ numerous frequency channels in most locations. The lower part of the L-band presents the most promising results, as LDACS A2A can operate there in any considered location. In fact, the lowest frequencies can be used anywhere in the considered region, which might allow LDACS A2A to have a globally available frequency channel for its operation.
\end{abstract}

Index Terms-LDACS, A2A, Frequency Planning, Cell Planning, DME, TACAN, L-band

\section{INTRODUCTION}

A IR traffic management (ATM) is being modernized worldwide, e.g., under the SESAR [1] framework in Europe and NextGen [2] in the USA, to sustain air traffic growth by providing new services and operational concepts. Some of these services require the availability of an air-to-air (A2A) data link capable of exchanging data directly between aircraft. As discussed in [3], currently available A2A data links are already reaching saturation and are not expected to be capable of supporting the new data intensive applications and concepts. These are to be supported by the extension towards A2A communications of the L-band digital aeronautical communications system (LDACS). Whilst the LDACS air-ground (A/G) component has already been developed [4], demonstrated in flight trials [5], and is currently undergoing the standardization process within the International Civil Aviation Organization
(ICAO) [6], the LDACS A2A data link is currently being developed within the German national project IntAirNet cofunded under the German aviation research program LuFo ("Luftfahrtforschungsprogramm").

LDACS shall operate in the $960-1164 \mathrm{MHz}$ frequency band under co-primary aeronautical mobile (route) service $(A M(R) S)$ allocation. Since this part of the L-band is allocated on a primary basis to the aeronautical radio navigation service (ARNS), LDACS is only allowed to operate in this frequency band if the existing ARNS systems are not harmfully interfered. Given that some of these systems occupy most of the 960-1164 MHz frequency range, a careful frequency planning must be conducted for LDACS in order to find the frequency channels that LDACS can use in each region without affecting the proper operation of the ARNS systems. The feasibility of the frequency planning for LDACS A/G has been assessed in [7]. Based on the results, the $964-1010 \mathrm{MHz}$ and $1110-1156$ $\mathrm{MHz}$ frequency bands have been proposed for allocation to the LDACS A/G reverse and forward links, respectively.

In this paper, we propose a frequency planning methodology for LDACS A2A, that can also be applied to the LDACS $\mathrm{A} / \mathrm{G}$ reverse link, and that yields no interference towards the ARNS systems operating in the L-band. We apply the proposed methodology to assess the feasibility of the frequency planning for LDACS A2A in the L-band under the constraint of avoiding harmful interference to legacy L-band systems. This work contributes to the development of LDACS A2A by analyzing the possible frequency bands where LDACS A2A might operate in the region of interest. After assessing how different operating parameters influence the spectrum availability of LDACS A2A, we derive recommendations for its design.

The paper is structured as follows. In Section II, we describe the LDACS communications system, including the A/G and A2A components, as well as the legacy systems operating in the L-band that have to be taken into account in the LDACS A2A frequency planning. We discuss the methodology for the frequency planning in Section III, including the mathematical model, the compatibility criteria, and the databases employed in our analysis. The results of the frequency planning are shown in Section IV and the conclusions are drawn in Section V. 


\section{LDACS A/G AND A2A}

LDACS is a cellular point-to-multipoint communications system, where each ground station (GS) serves airborne stations (ASs) located within a certain volume of space called cell [4]. Once the AS is connected to a GS, it gains access to the aeronautical telecommunication network (ATN) and becomes capable of communicating with other ATN users, such as air traffic control centers or other ATM entities.

LDACS is limited to regions where the GSs can be deployed. Consequently, it will not be possible to give direct support to aircraft operating in oceanic, remote, and polar (ORP) regions in its current state. This is to be solved by the LDACS extension towards A2A communications, hereinafter denoted as LDACS A2A, which shall extend the coverage of LDACS towards ORP regions by using intermediate ASs as relays. In other words, LDACS A2A will allow ASs to communicate directly with each other and, if needed, to relay messages towards and from the GSs. This way, even ASs operating in regions without ground infrastructure will have access to the ATN network by using both LDACS A2A and LDACS A/G components of LDACS. In fact, one of the main objectives in the design of LDACS A2A is to extend the LDACS coverage to the aircraft flying in the North Atlantic Corridor between North America and Europe.

Altogether, LDACS A2A could be seen as a communications, navigation, and surveillance (CNS) system. In addition to extending the LDACS A/G coverage, there are many other applications for LDACS A2A. First, LDACS A2A can be used for any application requiring direct communications between aircraft. For example, aircraft could use LDACS A2A to disseminate events and useful information, such as weather reports, among other aircraft, as well as to back up the flight recorder content on other aircraft, which would help reacting to aircraft incidents and clarifying them swiftly. Second, aircraft could navigate using LDACS A2A. For example, an AS could estimate its position by using pseudo-range multi-lateration from LDACS A2A transmissions from other aircraft with a good position estimation, e.g., derived from a Global Navigation Satellite System (GNSS) or from the LDACS A/G system. Third, LDACS A2A can be used for surveillance in an ADS-B (Automatic Dependent Surveillance - Broadcast) fashion. Each AS can broadcast useful information, such as identification, position, and velocity vectors. This information is picked up by neighbouring aircraft to be aware of its presence and act accordingly. This would allow aircraft to maintain a minimum separation with other aircraft and to coordinate trajectories with them.

LDACS A2A will operate in the 960-1164 MHz aeronautical frequency band following the World Radiocommunication Conference allocation for $\mathrm{AM}(\mathrm{R}) \mathrm{S}$ [8]. In order to communicate with other aircraft, an LDACS A2A station will require, at least, one frequency channel. Ideally, this channel would be available worldwide. Additional frequency channels might be used dynamically to achieve the data throughput required by the aforementioned applications. However, the 960-1164 MHz frequency band is already being employed by ARNS systems, including the distance measuring equipment (DME) and the tactical air navigation system (TACAN), both providing the slant range between an interrogating airborne station, i.e., the interrogator, and a responding ground station, i.e., the transponder. These systems employ frequency channels spaced $1 \mathrm{MHz}$ apart and spanning most of the aeronautical L-band. Thus, LDACS A2A might have to share a part of the spectrum with DME and TACAN. This is already done by LDACS A/G, which operates its reverse link in the 964-1010 MHz frequency band and its forward link in the $1110-1156 \mathrm{MHz}$ frequency band. LDACS A/G can still operate in the shared spectrum given that each DME/TACAN transponder only employs a pair of frequency channels to communicate with the interrogators in its coverage range. Thus, the frequency channels not used in a certain region might be employed, in principle, by the LDACS A/G stations deployed in that region. More specifically, LDACS A/G stations operating in a certain region will be able to use certain frequencies if minimum frequency and distance conditions with the DME/TACAN operations are guaranteed. The subset of frequency channels that can be used in each region, or location, is obtained by applying frequency planning ensuring no harmful interference towards the other systems. Given that LDACS A2A will, most likely, have to share spectrum with DME and TACAN systems, a similar frequency planning as the one conducted for LDACS A/G will have to be applied in order to guarantee the interference-free operation of all systems.

Although DME and TACAN are considered to be the main users of the aeronautical L-band, additional communications, navigation, and surveillance systems operate in this band and must be taken into account in the LDACS A2A frequency planning. Several surveillance systems operate at the 1030 $\mathrm{MHz}$ and $1090 \mathrm{MHz}$ frequencies, including the secondary surveillance radar (SSR), the traffic alert and collision avoidance system (TCAS), and the ADS-B service. In addition, although it is not used broadly, the universal access transceiver (UAT) system operates at $978 \mathrm{MHz}$. Another user of the Lband is the JTIDS/MIDS military communications system, which uses pseudo-random frequency hopping to quickly switch among 51 frequency channels distributed in three subbands between $969 \mathrm{MHz}$ and $1206 \mathrm{MHz}$ [9]. In addition, in order to protect the radionavigation satellite service (RNSS) operating in the upper part of the L-band, the ITU Resolution 417 restricts the maximum equivalent isotropic radiated power (EIRP) that an $\mathrm{AM}(\mathrm{R}) \mathrm{S}$ system can employ in the 960-1164 $\mathrm{MHz}$ frequency band [10].

In this work, we first define and conduct a frequency planning yielding the frequency channels that can be used by LDACS A2A without affecting the operation of DME and TACAN. Then, we show and analyze the results of the frequency planning for different candidate LDACS A2A frequency bands, taking into account the other systems operating in the L-band and the ITU Resolution 417. 


\section{FReQUency Planning}

The objective of the frequency planning for LDACS is to find a frequency, or a set of frequencies, that can be used by the LDACS stations in each region without any interference with the DME/TACAN operation. As different contributions have shown that LDACS can cope with DME/TACAN interference if suitable interference mitigation schemes are employed [11], our frequency planning only focuses on finding the frequencies that yield no interference from the LDACS stations towards DME/TACAN. To simplify the notation, we only refer to DME hereinafter, although we equally consider TACAN.

The frequency planning conducted in this work is based on the cell planning strategy defined for LDACS $A / G$ in [7]. In contrast to the work conducted in [7], where mainly the LDACS transmissions from LDACS ground stations were taken into account, we consider the transmissions from LDACS airborne stations, i.e. from the aircraft. Therefore, our strategy can be applied to the frequency and cell planning of LDACS A2A and of the LDACS A/G reverse link.

\section{A. Compatibility Criteria}

Our strategy is based on the fact that DME and LDACS can operate simultaneously and in the same region if the desiredto-undesired signal power ratio, hereinafter denoted as $\mathrm{D} / \mathrm{U}$ ratio, is maintained in any case above a certain $\mathrm{D} / \mathrm{U}$ compatibility threshold. If we consider the interference from LDACS towards DME, then our frequency planning has to guarantee that the D/U ratio (in this case, DME-to-LDACS signal power ratio) at any DME receiver will always stay above the $\mathrm{D} / \mathrm{U}$ compatibility threshold. This threshold can be obtained by conducting compatibility measurements between DME and LDACS, and generally varies with the frequency separation between the DME and LDACS transmissions. Let us denote the measured D/U threshold for compatibility between LDACS and DME as $D / U_{t h, T}\left(\Delta f_{T}\right)$ for DME transponders, and as $D / U_{t h, I}\left(\Delta f_{I}\right)$ for DME interrogators.

\section{B. Signal Propagation Model}

Let us first consider a wireless radio transmission at a frequency $f$ between two stations separated by a distance $d$. The signal transmitted by one station is received by the other station with a signal power denoted by $P_{r x}$, which can be obtained as

$P_{r x}[\mathrm{dBW}]=E I R P[\mathrm{dBW}]-L_{p}(d, f)[\mathrm{dB}]+G_{r x}[\mathrm{dBi}]-L_{r x}[\mathrm{~dB}]$,

where the EIRP is obtained as

$$
E I R P[\mathrm{dBW}]=P_{t x}[\mathrm{dBW}]+G_{t x}[\mathrm{dBi}]-L_{t x}[\mathrm{~dB}],
$$

and $L_{p}(d, f)$ represents the path losses for a distance $d$ and a signal frequency $f . G_{t x}$ and $G_{r x}$ denote the gains of the transmitting and receiving antennas, respectively, and $L_{t x}$ and $L_{r x}$ represent the losses between the radio and the antenna of the transmitter and of the receiver, respectively. Assuming free-space signal propagation and radio line-of-sight (LOS) between transmitter and receiver, we can estimate the path losses as

$L_{p, l o s}[\mathrm{~dB}]=20 \log _{10}(d[\mathrm{~km}])+20 \log _{10}(f[\mathrm{MHz}])+32.45 \mathrm{~dB}$.

However, given the earth curvature, long-range communications are generally blocked by the earth from a certain distance known as radio horizon. The radio horizon $r_{h}\left(h_{t x}, h_{r x}\right)$ depends on the altitude of the transmitter, i.e., $h_{t x}$, and of the receiver, i.e., $h_{r x}$, and can be estimated as

$$
r_{h}[\mathrm{~km}]=130.4\left(\sqrt{h_{t x}[\mathrm{~km}]}+\sqrt{h_{r x}[\mathrm{~km}]}\right),
$$

assuming a earth radius of $6378.137 \mathrm{~km}$ and scaling it by a factor of $4 / 3$ to account for the signal refraction in the atmosphere. From the radio horizon, a signal attenuation of $1.6 \mathrm{~dB}$ per nautical mile (NMi), i.e., $a=1.6 \mathrm{~dB} / \mathrm{NMi}$, can be expected in the L-band. Therefore, we can obtain the path losses $L_{p}\left(d, f, h_{t x}, h_{r x}\right)$ more generally as

$$
L_{p}[\mathrm{~dB}]=\left\{\begin{array}{ll}
L_{p, \operatorname{los}}(d, f) & \text { if } d \leq r_{h} \\
L_{p, \operatorname{los}}\left(r_{h}, f\right)+a \cdot\left(d-r_{h}\right) & \text { if } d>r_{h}
\end{array} .\right.
$$

Considering now the interference from LDACS towards DME, we can obtain the $\mathrm{D} / \mathrm{U}$ ratio of a DME communication interfered by an LDACS transmission as

$$
D / U[\mathrm{~dB}]=D[\mathrm{dBW}]-U[\mathrm{dBW}],
$$

where $D$ and $U$ can be obtained using (1) for DME and LDACS parameters, respectively. The $\mathrm{D} / \mathrm{U}$ ratio is obtained slightly differently for the DME transponders and the DME interrogators.

\section{Location of DME Transponders and Interrogators}

In order to conduct the frequency planning, the geographical location of the DME transponders is required, as well as some parameters of the DME transponders, such as the employed frequency channels, EIRP, and coverage region. This information is publicly available for some regions of the world. For example, the ICAO updates regularly the so-called COM3Table, which contains this information for a part of Europe.

In contrast with the DME transponders, the DME interrogators are installed in the aircraft and can move arbitrarily. Therefore, their position cannot be predicted in advance but a worst-case position must be considered in the frequency planning. Following our approach based on the $\mathrm{D} / \mathrm{U}$ ratio, the worst-case location of the DME interrogator will be the position where it is affected by the lowest $\mathrm{D} / \mathrm{U}$ ratio.

\section{Obtaining the D/U Ratio for DME Transponders}

In this case, we consider that a DME transponder at an altitude $h_{T}$ is receiving a transmission from a DME interrogator at a frequency $f_{T}$. In addition, it receives simultaneously an LDACS transmission at a frequency $f_{L}$ from an LDACS station at an altitude $h_{L}$. The distance $d_{L}$ between the DME transponder and the LDACS station can be obtained easily, as the positions of both stations are known. The position of the 
LDACS station and its transmit frequency are input parameters for the frequency planning.

Using (6), we can obtain the D/U ratio for DME transponders using a DME signal power of $D=-96 \mathrm{dBm}$. This is the minimum signal power at which long-range DME transponders must still be able to reply to an interrogation [7]. The power of the LDACS signal is obtained as

$$
U_{T}[\mathrm{dBm}]=E I R P_{L}[\mathrm{dBm}]-L_{p}[\mathrm{~dB}]+G_{T}[\mathrm{dBi}]-L_{T}[\mathrm{~dB}],
$$

where $E I R P_{L}$ represents the EIRP of the LDACS station, $L_{p}$ the path losses, $G_{T}$ is the DME antenna gain at the direction of arrival of the LDACS transmission, and $L_{T}$ represents the losses between the antenna and the radio of the receiving DME station. Whilst $L_{p}$ is obtained using (5), we assume a maximum antenna gain $G_{T}=9.1 \mathrm{dBi}$ [7] and no losses, i.e., $L_{T}=0 \mathrm{~dB}$, which are conservative assumptions in favor of the DME system.

The LDACS station can operate without affecting the operation of the DME transponder if

$$
D / U_{T}=-96-U_{T}[\mathrm{dBm}] \geq D / U_{t h, T}\left(\Delta f_{T}\right)+\zeta
$$

is fulfilled, where $\Delta f_{T}=f_{T}-f_{L}$, and $\zeta$ is a safety margin considered for the frequency planning. We use $\zeta=10 \mathrm{~dB}$ in our analysis.

\section{E. Obtaining the D/U Ratio for DME Interrogators}

In this case, we consider a DME interrogator receiving a transmission at a frequency $f_{I}$ from a DME transponder. The DME interrogator is located within the coverage range of the DME transponder, at an altitude $h_{I}$ and a distance $d_{I}$ to the transponder. The LDACS station transmits at a frequency $f_{L}$ and flies at an altitude $h_{L}$. The distance between the LDACS station and the DME interrogator is denoted by $d_{L}$.

The $\mathrm{D} / \mathrm{U}$ ratio can be obtained as

$$
\begin{array}{r}
D / U_{I}=\left(E I R P_{T}-L_{p}\left(d_{I}, f_{I}\right)+G_{I}-L_{I}\right) \\
-\left(E I R P_{L}-L_{p}\left(d_{L}, f_{L}\right)+G_{I}-L_{I}\right) \\
=E I R P_{T}-E I R P_{L}-\left(L_{p}\left(d_{I}, f_{I}\right)-L_{p}\left(d_{L}, f_{L}\right)\right),
\end{array}
$$

where we assume that the DME interrogator has the same antenna gain and receiver losses for both signals. If the interrogator is also within the LOS of the LDACS station, the $\mathrm{D} / \mathrm{U}$ ratio simplifies to

$D / U_{I}=E I R P_{T}-E I R P_{L}-20 \log _{10}\left(\frac{f_{I}}{f_{L}}\right)-20 \log _{10}\left(\frac{d_{I}}{d_{L}}\right)$.

We have to guarantee that no DME interrogator within a certain DME cell is affected by the LDACS operation at a certain frequency and location. Thus, we obtain the minimum $\mathrm{D} / \mathrm{U}$ ratio that a DME interrogator can experience anywhere in the cell, and compare it with the minimum D/U ratio required for compatibility. This could be done by obtaining the $\mathrm{D} / \mathrm{U}$ ratio for each position in the cell. However, it can be simplified by distinguishing two cases depending on the location of the LDACS station. First, when the LDACS station is outside the $\mathrm{DME}$ cell, the minimum $\mathrm{D} / \mathrm{U}$ ratio is found when the $\mathrm{DME}$ interrogator is on the DME cell border closest to the LDACS station. The exact location on the border can be found by obtaining the $\mathrm{D} / \mathrm{U}$ ratio for different altitudes $h_{I}$ between the minimum altitude $h_{I, \min }$ and the maximum altitude $h_{D M E}$ considered. We use $h_{I, \min }=1000$ feet in our analysis. The maximum altitude $h_{D M E}$ is given by the maximum coverage altitude of each DME cell. Second, when the LDACS station is inside the DME cell, the minimum aircraft separation becomes the deciding factor. The minimum D/U ratio is observed in the close vicinity of the LDACS station at the position maximizing $d_{I} / d_{L}$ but still guaranteeing the minimum horizontal and vertical separation, i.e., $\Delta d_{h}$ and $\Delta d_{v}$, respectively. Although the minimum separation between aircraft depends on the airspace regulations, we use $\Delta d_{h}=2.5 \mathrm{NMi}$ and $\Delta d_{v}=1000$ feet in our frequency planning as a simplification. Note that higher separations leading to less stringent interference conditions, i.e., higher minimum $\mathrm{D} / \mathrm{U}$ ratio, are expected in general.

The LDACS station can operate without affecting the operation of any DME interrogator operating in the DME cell if

$$
\min \left(D / U_{I}\right) \geq D / U_{t h, I}\left(\Delta f_{I}\right)+\zeta
$$

is fulfilled, where $\min \left(D / U_{I}\right)$ is the minimum $\mathrm{D} / \mathrm{U}$ ratio for any location within the DME cell, and $\Delta f_{I}=f_{I}-f_{L}$.

\section{F. DME-Compatible LDACS A2A Frequency Planning}

The operation of LDACS A2A at a certain location, i.e., coordinates and altitude, and using certain transmission parameters, i.e., EIRP and transmit frequency, is considered to be compatible with DME if (8) and (11) are satisfied for all DME transponders and interrogators. In order to evaluate this condition, we first load the information of the DME transponders, including their location, i.e., coordinates and altitude, coverage range $R_{D M E}$, coverage altitude $h_{D M E}$, EIRP, and frequency channels. Then, we iterate over each transponder and evaluate (8) and (11). If both conditions are fulfilled for all DME transponders and, consequently, for all DME interrogators, the LDACS position and transmission parameters are compatible with DME. We then iterate over all the positions and transmission parameters of the LDACS station that we want to evaluate.

\section{G. DME/TACAN Database and D/U Thresholds for Compati-} bility

The DME/TACAN information used for our frequency planning is extracted from different sources. For Europe, we use the so-called COM3 table officially published by ICAO, which contains the information of the pan-European DME and TACAN transponders, including their coordinates, EIRPs, frequency channels, and coverage ranges and altitudes. For North America, however, we have not found a publicly available official DME/TACAN database. In order to conduct this frequency planning, the FAA provided us with a list of DME transponders deployed in North America, including their coordinates, EIRPs, frequency channels, and coverage ranges and altitudes. For Canada, we consider the DME transponders listed in [12]. Given that only the coordinates and frequency 


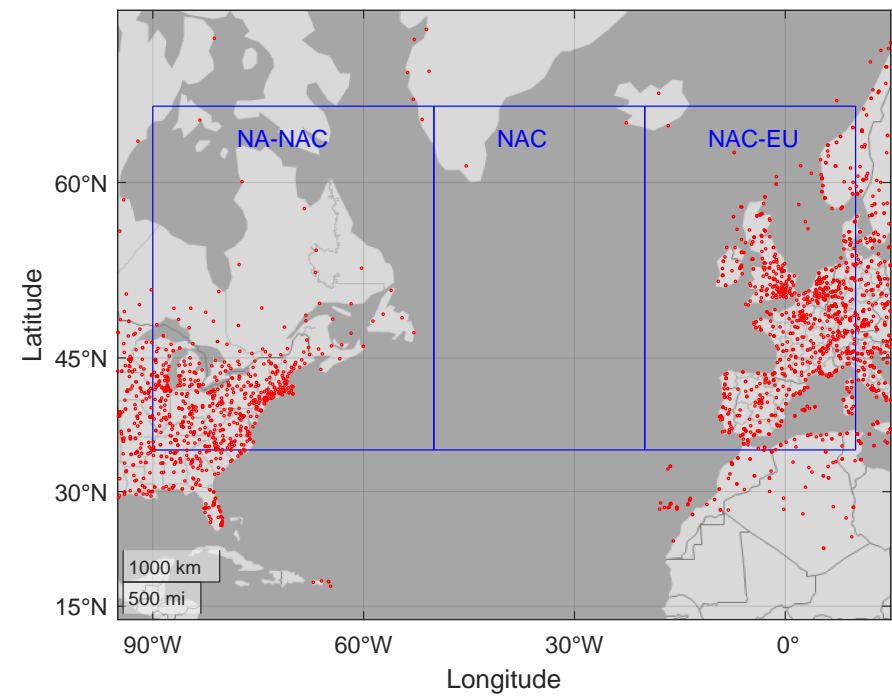

Fig. 1: DME/TACAN transponders (red dots) and region of interest (labelled blue rectangles) considered for the LDACS A2A frequency planning.

channels are provided in [12], we assume that the DME transponders cover a range of $130 \mathrm{NMi}$ up to an interrogator altitude of 60000 feet with an EIRP of 100W. We additionally found in [13] a list of the DME and TACAN transponders deployed in Greenland, including their coordinates, frequency channels, and coverage ranges and altitudes. As no EIRP was provided, we consider 100W EIRP as a conservative assumption in favor of DME/TACAN. In addition, we assume $h_{T}=10 \mathrm{~m}$ for all DME/TACAN stations. Note that, as only a part of the DME/TACAN information has been extracted from an official database, this frequency planning should not be considered as final but as a best effort to assess the feasibility of a frequency planning for LDACS A2A.

We show in Fig. 1 the position of the DME and TACAN transponders considered in our frequency planning, as well as the three segments comprising the region of interest for our LDACS A2A frequency planning. Note that the DME and TACAN transponders located outside the region of interest are also taken into account in the frequency planning. Fig. 2 depicts the number of DME/TACAN transponders using a certain frequency for transmissions (Fig. 2a) and for receptions from DME/TACAN interrogators (Fig. 2b). As expected, most of the aeronautical L-band is used by DME/TACAN stations. In practice, only the lowest frequencies, i.e., between 960 and $978 \mathrm{MHz}$, and the frequencies around $1030 \mathrm{MHz}$ and 1090 $\mathrm{MHz}$, allocated to surveillance systems, are not widely used by DME/TACAN.

The minimum $\mathrm{D} / \mathrm{U}$ signal power ratio required by DME/TACAN to operate correctly despite the LDACS A/G operation was estimated in several preliminary measurements conducted in the laboratory and presented in [14]. Given that no compatibility measurements between LDACS A2A and DME/TACAN are still available, and that the LDACS A2A transmissions are expected to be very similar to the

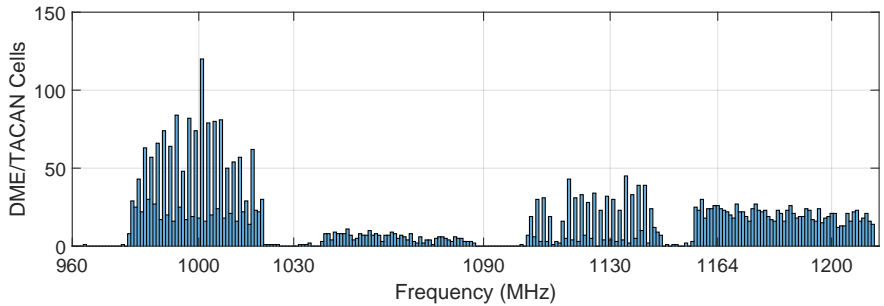

(a) Transmit frequencies of DME/TACAN transponders.

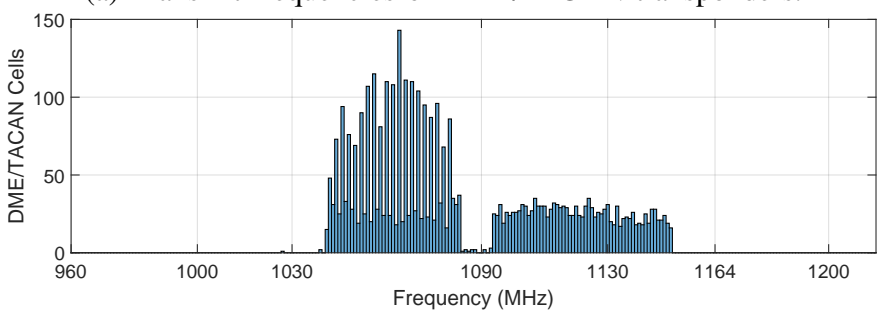

(b) Transmit frequencies of DME/TACAN interrogators.

Fig. 2: Number of considered DME/TACAN transponders using each frequency for transmissions (Fig. 2a) and receptions from interrogators (Fig. 2b).

transmissions in the LDACS A/G reverse link, we use the obtained measurement results as basis for our analysis. In addition to the LDACS A/G transmissions, these measurements also considered the background interference generated by other DME/TACAN and JTIDS/MIDS stations. Unfortunately, the evaluation of the measurement data showed that the background interference (without LDACS) used in the measurements had been unrealistically overdimensioned and could, in fact, disrupt the DME/TACAN operation even without LDACS. In addition, some limitations in the measurement setup prevented the analysis from yielding conclusive results for the low signal power domain. Consequently, the estimated minimum $\mathrm{D} / \mathrm{U}$ ratios were considered to be too conservative in favor of DME/TACAN and new measurements, with a refined compatibility criteria, a realistic background interference and more sensitive measurement equipment, were regarded as necessary. Given that less stringent D/U thresholds are expected from the future compatibility measurements between LDACS and DME, we employ the preliminary minimum D/U signal power ratios to assess the feasibility of the LDACS A2A frequency planning even under unrealistically strict conditions.

Table I contains the preliminary minimum $\mathrm{D} / \mathrm{U}$ ratio $D / U_{I, t h}$ required by the DME/TACAN interrogators to operate correctly. These values are based on the compatibility tests conducted between the reverse link of LDACS A/G and the receiving DME/TACAN interrogators. Note that the D/U threshold depends on the frequency separation $\Delta f$ between the LDACS and DME/TACAN signals. In addition, one can see that the $\mathrm{D} / \mathrm{U}$ threshold decreases very slowly for $\Delta f \geq$ $2 \mathrm{MHz}$, which is partly caused by the low sensitivity of the employed measurement setup during the tests. For frequency separations higher or equal than $\Delta f_{\text {stop }}$, it is assumed that the LDACS transmissions do not affect the DME/TACAN opera- 
tion, regardless of the $\mathrm{D} / \mathrm{U}$ signal power ratio. Unfortunately, $\Delta f_{\text {stop }}$ could not be measured in the conducted compatibility tests because of the limiting measurement setup. Therefore, we assume $\Delta f_{\text {stop }}=20 \mathrm{MHz}$ in our frequency planning.

Given that the maximum frequency of the LDACS A/G reverse link is $1010 \mathrm{MHz}$, and we can see in Fig. 2b that the minimum receive frequency of the DME/TACAN transponders is much higher, i.e., above $1030 \mathrm{MHz}$, no compatibility measurements were conducted between the LDACS A/G reverse link and the DME/TACAN transponders as receivers. Consequently, we employ for our frequency planning the results of the compatibility measurements between the LDACS A/G forward link and the receiving DME/TACAN transponders. The preliminary minimum $\mathrm{D} / \mathrm{U}$ ratio $D / U_{T, t h}$ required by the receiving DME/TACAN transponders to operate correctly can be seen in Table II.

Considering that the employed minimum D/U signal power ratios are derived from the compatibility measurements between LDACS A/G and DME/TACAN, and that the conducted measurements have been regarded as unrealistically challenging for DME/TACAN, our frequency planning should not be regarded as final but as a first analysis of the feasibility of the LDACS A2A frequency planning. Nevertheless, the frequency planning methodology proposed in this paper can be applied to conduct the final LDACS A2A frequency planning when conclusive $\mathrm{D} / \mathrm{U}$ thresholds and an official DME/TACAN database are available.

\section{$H$. Compatibility with other systems operating in the L-band}

Unfortunately, no compatibility criteria have been defined yet between LDACS and the systems operating at $1030 \mathrm{MHz}$ and $1090 \mathrm{MHz}$, such as SSR. However, it can be assumed that a minimum frequency separation between those frequencies and the LDACS A2A frequencies should suffice to guarantee the compatibility. In fact, we can see that the frequency bands allocated to LDACS A/G are separated by, at least, $20 \mathrm{MHz}$ to the $1030 \mathrm{MHz}$ and $1090 \mathrm{MHz}$ frequencies. If the same criteria is applied to LDACS A2A, we can expect LDACS A2A not to be allowed to operate between $1010 \mathrm{MHz}$ and $1050 \mathrm{MHz}$, and between $1070 \mathrm{MHz}$ and $1110 \mathrm{MHz}$. A similar approach could be expected for UAT, which operates at $978 \mathrm{MHz}$. In this case, however, the UAT operating frequency falls within the LDACS reverse link frequency band and no minimum frequency separation can be inferred from it.

Although the impact of LDACS A/G on JTIDS/MIDS has been assessed in [9], no compatibility criteria between LDACS A/G and JTIDS/MIDS have been defined so far. In addition, we have not found any information regarding either the location of fixed JTIDS/MIDS stations, or the national restrictions limiting the use of certain frequency channels. Consequently, we do not take JTIDS/MIDS into account in our frequency planning.

In addition, the ITU Resolution 417 safeguards the operation of the RNSS receivers by restricting the maximum EIRP that an $\mathrm{AM}(\mathrm{R}) \mathrm{S}$ system can use at certain frequencies. Given that the ITU Resolution 417 does not specify the maximum EIRP

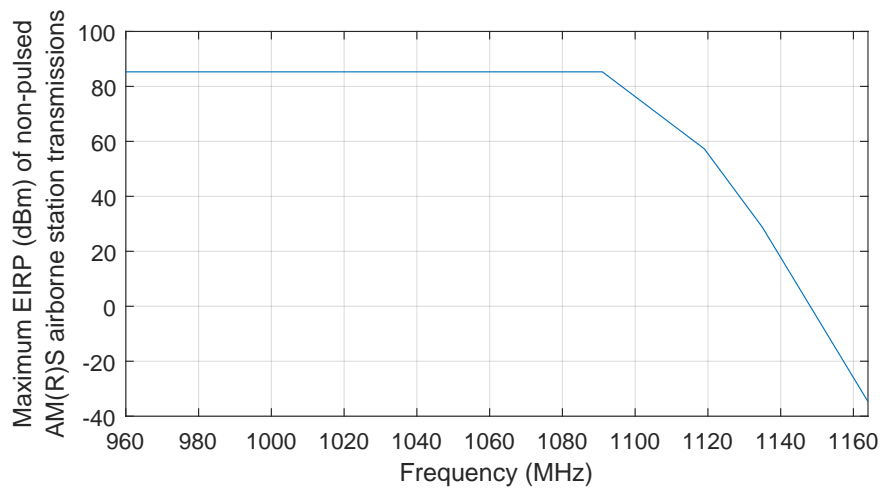

Fig. 3: Maximum EIRP of AMR(S) non-pulsed transmissions in the 960-1164 MHz frequency band according to the ITU Resolution 417 [10].

for pulsed transmissions, as the ones expected for LDACS A2A, we consider the maximum EIRP shown in Fig. 3 for non-pulsed transmissions as a worst-case assumption. Thus, for an EIRP of $41 \mathrm{dBm}$, the highest frequency that LDACS A2A would be allowed to use is $1128.1 \mathrm{MHz}$ in the worst case. In order to have a more complete view of the spectrum availability for LDACS A2A, we first conduct the frequency planning without considering the ITU Resolution 417, and then take it into account in the discussion of the results and in the definition of the candidate frequency bands for LDACS A2A.

\section{RESULTS}

We focus our analysis on the North Atlantic Corridor and its connecting American and European territories, i.e., northeast coast of North America and western Europe. We consider a region of interest limited by a longitude between $90^{\circ} \mathrm{W}$ and $10^{\circ} \mathrm{E}$, and a latitude between $35^{\circ} \mathrm{N}$ and $65^{\circ} \mathrm{N}$. We divide this region into the three segments shown in Fig. 1: NA-NAC for longitudes between $90^{\circ} \mathrm{W}$ and $50^{\circ} \mathrm{W}, N A C$ for longitudes between $50^{\circ} \mathrm{W}$ and $20^{\circ} \mathrm{W}$, and $N A C-E U$ for longitudes between $20^{\circ} \mathrm{W}$ and $10^{\circ} \mathrm{E}$.

In order to conduct the frequency planning, we define the set of positions where the aircraft carrying the LDACS A2A radio can be located. We divide the region of interest into a latitude-longitude grid with $0.1^{\circ}$ steps. For each location, we consider different LDACS airborne station altitudes $h_{L}=(1,3,6,9,12,15,18) \mathrm{km}$. As the frequencies to be used by LDACS A2A have not been decided yet, we initially assume that it might operate anywhere in the aeronautical L-band between 960 and $1164 \mathrm{MHz}$. In addition, we consider a $0.5 \mathrm{MHz}$ frequency grid as the one considered for LDACS A/G. Thus, the candidate frequency channels for the LDACS A2A frequency planning are centered at $f_{L}=$ $(960,960.5,961, \ldots, 1163.5,1164) \mathrm{MHz}$. We initially assume an LDACS A2A EIRP of $41 \mathrm{dBm}$, i.e., EIRP $P_{L}=41 \mathrm{dBm}$, which is the expected value given in the LDACS specification [4] for the LDACS airborne station.

We conduct the frequency planning described in Section III for the entire region of interest and show the results for each 
TABLE I: Considered D/U threshold for receiving DME/TACAN interrogators [14].

\begin{tabular}{|c|c|c|c|c|c|c|c|c|c|}
\hline$|\Delta f|[\mathrm{MHz}]$ & 0 & 0.5 & 1 & 2 & 3 & 4 & 5 & 10 & $\begin{array}{c}\geq 14< \\
\Delta f_{\text {stop }}\end{array}$ \\
\hline$D / U_{I, t h}[\mathrm{~dB}]$ & 15 & 1 & -20 & -45 & -49 & -47 & -45 & -50 & -52 \\
\hline
\end{tabular}

TABLE II: Considered D/U threshold for receiving DME/TACAN transponders [14].

\begin{tabular}{|c|c|c|c|c|c|c|c|c|c|c|c|}
\hline$|\Delta f|[\mathrm{MHz}]$ & 0 & 0.5 & 1 & 1.5 & 2 & 2.5 & 3 & 4 & 5 & 6 & $\begin{array}{c}\geq 7< \\
\Delta f_{\text {stop }}\end{array}$ \\
\hline$D / U_{T, t h}[\mathrm{~dB}]$ & 13 & 16 & 6 & -2 & -5 & -9 & -14 & -30 & -41 & -52 & -57 \\
\hline
\end{tabular}

one of the three segments separately. We first analyse the percentage of locations where each candidate frequency channel can be used at the different altitudes of the LDACS A2A transmitter. Fig. 4 shows the obtained results for the NAC-EU segment defined in Fig. 1. One can see that some frequencies, approximately between $1040 \mathrm{MHz}$ and $1156 \mathrm{MHz}$, can only be used in some locations at certain aircraft altitudes. In general, less frequencies are available for high-flying LDACS A2A stations, which can be explained by their higher radio LOS (see (4)). As expected from Fig. 2, the frequencies around 1030 $\mathrm{MHz}$ and $1090 \mathrm{MHz}$ yield practically no interference towards DME/TACAN. However, these frequencies are allocated to surveillance systems and a separate compatibility analysis should be conducted to assess whether LDACS A2A can operate around those frequencies. Likewise, we can see a small group of frequencies in the higher part of the L-band, i.e., around $1154 \mathrm{MHz}$, where LDACS A2A could operate in most regions without affecting DME/TACAN. However, this operation might not be feasible because of the ITU Resolution 417. The most interesting frequency band where LDACS A2A could operate in the $N A C-E U$ region is the lower Lband, i.e., between $960 \mathrm{MHz}$ and $1040 \mathrm{MHz}$, for multiple reasons. First, these frequencies are usable in more locations compared to higher frequencies. Second, the altitude of the LDACS A2A station does not affect significantly the number of locations where a frequency can be used. Third, it would be in line with the LDACS A/G allocation for the airborne station transmissions, i.e., 964-1010 MHz. Fourth, the 33 frequency channels between $960 \mathrm{MHz}$ and $976 \mathrm{MHz}$ can be used in more than $95 \%$ of the considered locations and, most importantly, the 15 frequency channels between $960 \mathrm{MHz}$ and $967 \mathrm{MHz}$ can be used anywhere in the $N A C-E U$ region. These results are crucial for LDACS A2A, as they show that there is a subset of frequencies that do not require any further coordination with DME/TACAN, at least in the NAC-EU region and under the parameters of our analysis.

Fig. 5 shows the results of the frequency planning for the NA-NAC region (see Fig. 1). Comparing Fig. 4 and Fig. 5, we see that the results for both regions are generally quite similar. However, we observe that most frequencies, with the exception of the frequencies above $1137 \mathrm{MHz}$, are usable in

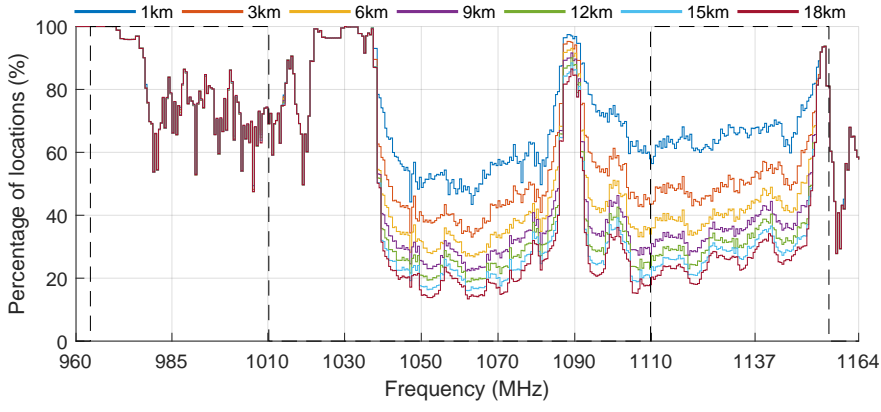

Fig. 4: Percentage of locations in the $N A C-E U$ region where each frequency can be used by LDACS A2A without interfering the DME/TACAN operation. The different lines show the results for different LDACS A2A altitudes $h_{L}$. The black dashed line defines the LDACS A/G frequency bands.

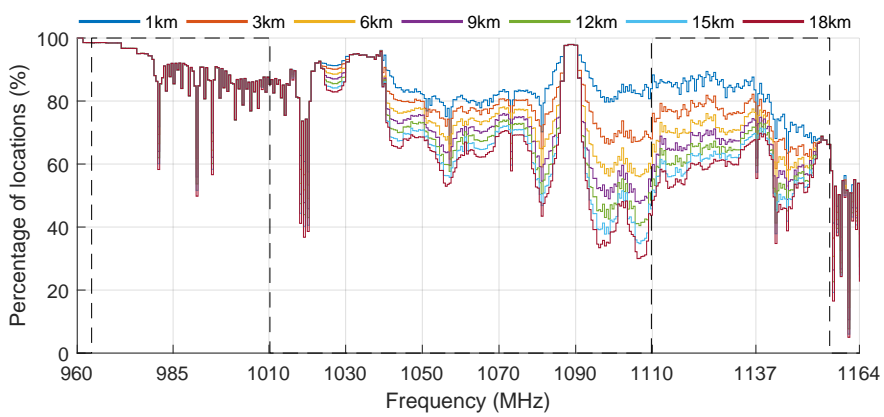

Fig. 5: Percentage of locations in the $N A-N A C$ region where each frequency can be used by LDACS A2A without interfering the DME/TACAN operation. The different lines show the results for different LDACS A2A altitudes $h_{L}$. The black dashed line defines the LDACS A/G frequency bands.

more locations in the NA-NAC region than in the NAC-EU region. Again, the most promising frequency band for LDACS A2A is the lower part of the L-band between $960 \mathrm{MHz}$ and $1040 \mathrm{MHz}$. In fact, the 37 frequency channels between 960 and $978 \mathrm{MHz}$ can be used in more than $95 \%$ of the NA-NAC region. Moreover, the three frequency channels between 960 $\mathrm{MHz}$ and $961 \mathrm{MHz}$ can be used anywhere.

Fig. 6 shows the results of our frequency planning for the 


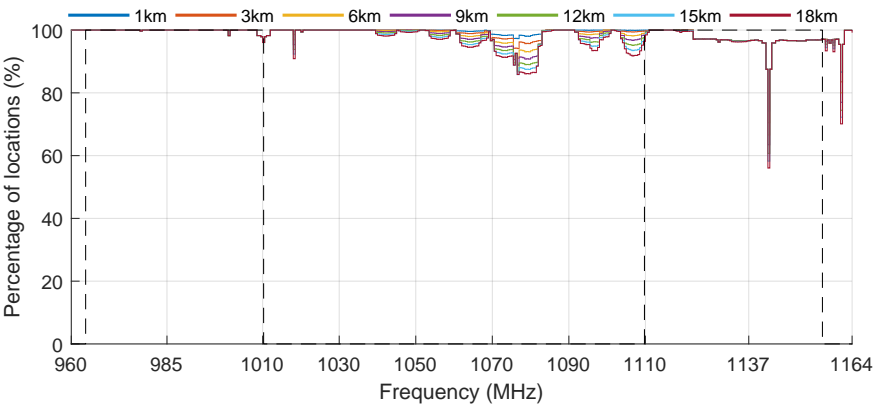

Fig. 6: Percentage of locations in the $N A C$ region where each frequency can be used by LDACS A2A without interfering the DME/TACAN operation. The different lines show the results for different LDACS A2A altitudes $h_{L}$. The black dashed line defines the LDACS A/G frequency bands.

North Atlantic Corridor, i.e., the $N A C$ region (see Fig. 1). As expected because of the absence of DME/TACAN stations operating in the ocean, most frequencies can be used anywhere in the $N A C$ region without affecting the operation of DME/TACAN. In fact, practically all frequency channels up to $1040 \mathrm{MHz}$ are usable throughout the $N A C$ region, and only a few frequencies are restricted in some locations close to the mainland.

Interestingly, the reverse link of LDACS A/G starts from a frequency of $964 \mathrm{MHz}$. However, we see in the results shown in Fig. 4-6, that the lower frequencies, i.e., 960-964 $\mathrm{MHz}$, could be of great interest for the operation of LDACS A2A. These frequencies present the lowest impact towards DME/TACAN and they could be used almost everywhere within the entire region of interest. In fact, an LDACS A2A operation in a globally usable frequency channel might be possible at these frequencies. However, an LDACS A2A operation at the lowest frequencies might require to develop additional compatibility criteria between LDACS A2A and the mobile communication systems operating below $960 \mathrm{MHz}$. In addition, the importance of the lower part of the L-band for LDACS A2A stresses the need for specific compatibility criteria between LDACS A2A and UAT operating at $978 \mathrm{MHz}$.

In order to have a more complete view of the results of the LDACS A2A frequency planning for the entire region of interest, we now depict in Fig. 7 the number of frequency channels, between $960 \mathrm{MHz}$ and $1164 \mathrm{MHz}$ in steps of 0.5 $\mathrm{MHz}$, that can be used by LDACS A2A without affecting the operation of DME/TACAN. For each location, we only consider the frequency channels that can be used at all considered altitudes, i.e., in the worst case. We can see in Fig. 7 the significant difference in the number of available frequency channels between the mainland regions and the North Atlantic Corridor, where most frequency channels can be used. Thus, the total spectrum available for the LDACS A2A stations would reach a maximum in the North Atlantic Corridor and decrease gradually as the airborne stations approach the mainland. Additionally, we see that LDACS A2A will be able to use numerous frequency channels in the transition between

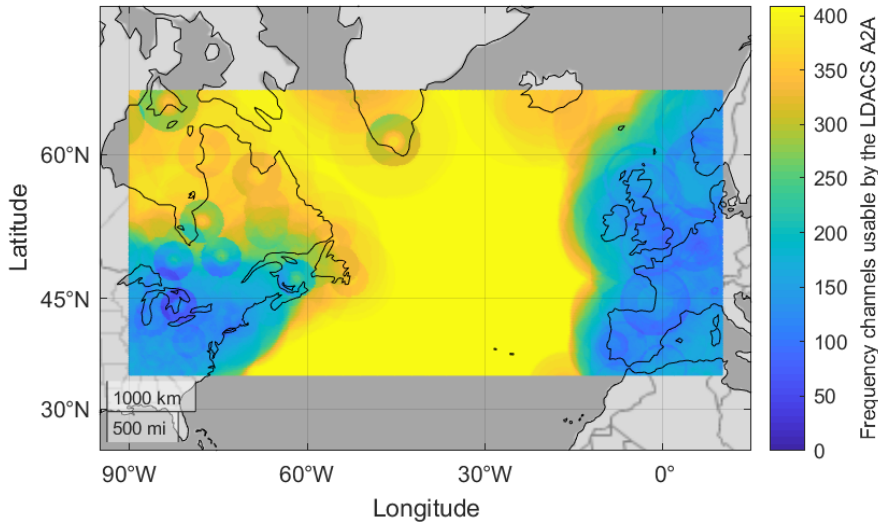

Fig. 7: Number of frequency channels between $960 \mathrm{MHz}$ and $1164 \mathrm{MHz}$, in steps of $0.5 \mathrm{MHz}$, that LDACS A2A can use without interfering the operation of DME/TACAN.

the oceanic and the continental airspace. This is an important result as LDACS A2A is expected to extend the LDACS $\mathrm{A} / \mathrm{G}$ coverage towards oceanic, remote, and polar regions, and therefore a high throughput might be required in the transition regions for the LDACS A2A stations to act as gateways for the LDACS ground stations. In the continental airspace, we see that LDACS A2A can still use a significant amount of frequency channels.

Logically, LDACS A2A will also have to be compatible with the other systems operating in the L-band. As discussed in Section III, we assume that a frequency separation of $20 \mathrm{MHz}$ between the LDACS A2A transmissions and the $1030 \mathrm{MHz}$ and $1090 \mathrm{MHz}$ frequencies is enough to guarantee the compatibility between LDACS A2A and the surveillance systems operating at those frequencies. In addition, we take into account the ITU Resolution 417 and assume that LDACS A2A might not be allowed to operate at a frequency above $1128.1 \mathrm{MHz}$ if an EIRP of $41 \mathrm{dBm}$ is used. These constraints are fulfilled if LDACS A2A operates in three discontiguous frequency bands: $960-1010 \mathrm{MHz}, 1050-1070 \mathrm{MHz}$, and 1110$1128 \mathrm{MHz}$. Fig. 8 shows the number of frequency channels that LDACS A2A can use without affecting the operation of DME/TACAN if we restrict the LDACS A2A transmissions to these three frequency bands. Similarly to Fig. 7, we see that the number of available frequency channels is maximum in the oceanic airspace and reduces gradually as the airborne stations approach the mainland. The airborne stations flying in continental airspace will still be able to use a significant amount of frequency channels, especially in Europe. In the $N A-N A C$ region, however, we find some locations where the number of frequency channels available for LDACS A2A is significantly lower than in other mainland regions. Fortunately, LDACS A2A can still employ at least 5 frequency channels in any considered location and altitude.

Considering now that LDACS A2A might only be able to operate in the frequency bands already allocated to LDACS $\mathrm{A} / \mathrm{G}$, we show in Fig. 9 the number of frequency channels available for LDACS A2A in the 964-1010 MHz and 1110- 


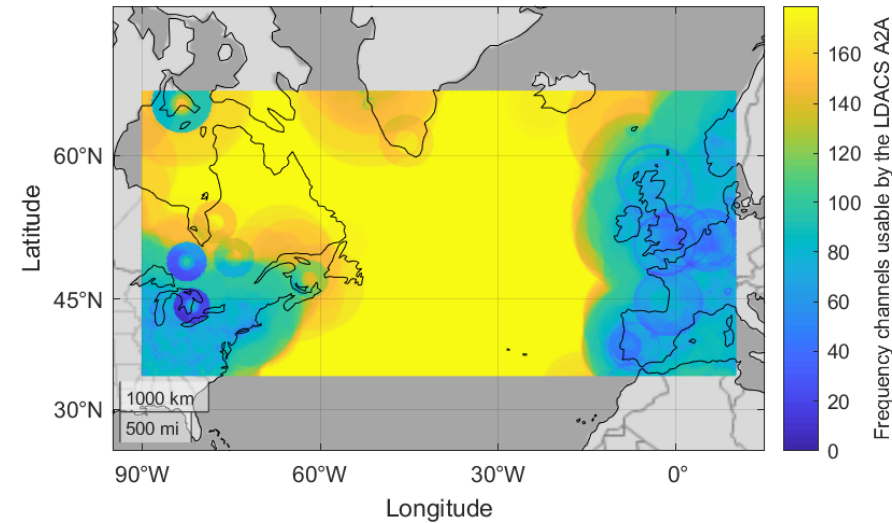

Fig. 8: Number of frequency channels that LDACS A2A can use without interfering the operation of DME/TACAN. In order to protect the operations at $1030 \mathrm{MHz}$ and 1090 $\mathrm{MHz}$ and to fulfill the ITU Resolution 417 for non-pulsed $\mathrm{AM}(\mathrm{R})$ airborne station transmissions with an EIRP of 41 $\mathrm{dBm}$, we only consider the frequency channels within three discontiguous frequency bands: $960-1010 \mathrm{MHz}, 1050-1070$ $\mathrm{MHz}$, and 1110-1128 MHz.

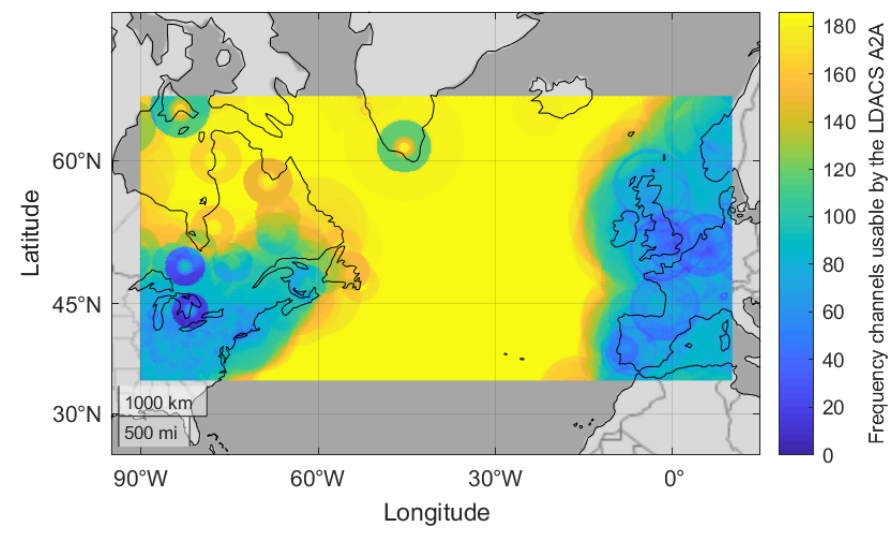

Fig. 9: Number of frequency channels in the LDACS A/G frequency bands, i.e, $964-1010 \mathrm{MHz}$ and $1110-1156 \mathrm{MHz}$, that LDACS A2A can use without interfering the operation of DME/TACAN.

$1156 \mathrm{MHZ}$ frequency bands. We still observe that LDACS A2A will be able to operate using numerous frequency channels in any considered region. Even in the most challenging locations, LDACS A2A can still operate using, at least, 2 frequency channels. These results are very important for LDACS A2A as they indicate that, even under the many worstcase assumptions made throughout our analysis, LDACS A2A can still find frequencies to operate in any considered location and altitude. In reality, a higher number of usable frequency channels are expected, especially in the most challenging regions.

Let us now assess how a different LDACS A2A EIRP affects the frequency planning. Fig. 10 shows the percentage of locations in the entire region of interest (including the $N A$ -

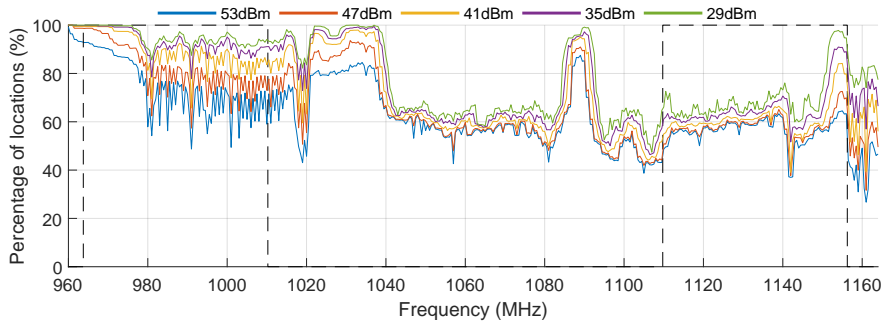

Fig. 10: Percentage of locations within the entire region of interest (including $N A-N A C, N A C$, and $N A C-E U$ regions) where each frequency can be used by LDACS A2A without interfering the DME/TACAN operation. The different lines show the results for different LDACS A2A EIRPs. The black dashed line defines the LDACS A/G frequency bands.

$N A C, N A C$, and $N A C-E U$ regions) where LDACS A2A can use the frequency channels in the $960-1164 \mathrm{MHz}$ frequency band at different EIRPs without affecting the operation of DME/TACAN. As expected, decreasing the EIRP increases the percentage of locations where a frequency channel can be used. In addition, we see that the frequencies below 1040 $\mathrm{MHz}$ generally profit more from the decrease in the EIRP than the higher frequencies. It is also important to notice that a decrease in the EIRP increases the number of frequency channels that can be used anywhere in the region of interest. This is very important for LDACS A2A as it indicates that the EIRP could be reduced in the most challenging locations to enable the LDACS A2A operation at certain frequencies of interest. Thus, we recommend LDACS A2A to be able to adjust its transmit power dynamically. This would allow LDACS A2A to profit from a higher EIRP, e.g., in order to increase its data throughput or communications range, in the less challenging regions, but to also be able to reduce the EIRP in order to use enough frequency channels in the most challenging regions.

Fig. 11 shows the minimum number of frequency channels available in a percentage of the locations for different LDACS A2A EIRPs and for different frequency bands. We can see in Fig. 11a the results for the 960-1010 MHz, 1050-1070 $\mathrm{MHz}$, and $1110-f_{\max } \mathrm{MHz}$ frequency bands, where $f_{\max }$ is the highest frequency fulfilling the ITU Resolution 417 for each EIRP (see Fig. 3). One can clearly see that a lower EIRP yields more candidate frequency channels and, in any case, more usable frequency channels. Moreover, we see that the minimum number of frequency channels available in any location is 5 for the expected LDACS A2A EIRP of $41 \mathrm{dBm}$. It increases up to 7 and 42 for an EIRP of $35 \mathrm{dBm}$ and $29 \mathrm{dBm}$, respectively, and decreases down to 3 and 0 for an EIRP of $47 \mathrm{dBm}$ and $53 \mathrm{dBm}$, respectively. These results indicate that the EIRP could be reduced in order to find more frequency channels where LDACS A2A could operate in the most challenging regions. In addition, the results in Fig. 11a show that LDACS A2A will still be able to operate even if its EIRP has to be increased up to $47 \mathrm{dBm}$, but that it will no longer be able to operate in some regions if a much higher 
EIRP of $53 \mathrm{dBm}$ is used.

Let us now limit the frequency planning to the LDACS A/G frequency bands. The minimum number of usable frequency channels for a percentage of the locations is shown in Fig. 11b for both LDACS A/G frequency bands combined, i.e., 964$1010 \mathrm{MHz}$ and 1110-1156 MHz. Comparing Fig. 11a and Fig. 11b, we see that the results for both frequency alocations are quite similar. However, an LDACS A2A operation with an EIRP of $47 \mathrm{dBm}$, which is feasible for the frequency bands considered in Fig. 11a, might not be supported in all locations if only the LDACS A/G frequency bands are considered. Importantly, LDACS A2A would still be able to use, at least, 2 frequency channels within the LDACS A/G frequency bands in any considered location if the expected EIRP of $41 \mathrm{dBm}$ is employed. The number of usable channels can still be effectively increased by decreasing the EIRP below $41 \mathrm{dBm}$. The results for the $964-1010 \mathrm{MHz}$ reverse link band and for the 1110-1156 MHz forward link band are shown separately in Fig. 11c and Fig. 11d, respectively. One can see that the 964-1010 MHz frequency band suffices for LDACS A2A to be able to operate at any considered location with the expected EIRP of $41 \mathrm{dBm}$. Moreover, many frequency channels are usable in most of the considered locations, e.g. at least 60 frequency channels in $90 \%$ of the locations. We also see that the EIRP could be reduced to effectively increase the number of channels usable in the most challenging regions. Note that these results are also very important for the LDACS A/G reverse link, as they indicate that it will be able to operate anywhere in the region of interest even without having to reduce its expected EIRP of $41 \mathrm{dBm}$. LDACS A2A could still operate in many locations if only the $1110-1156 \mathrm{MHz}$ frequency band is employed. However, this band would not suffice for LDACS A2A to operate anywhere in the considered region. These results highlight the importance of the lower part of the aeronautical L-band for the operation of LDACS A2A, especially in the continental regions as shown in Fig. 4 and Fig. 5.

\section{CONCLUSiON}

In this paper, we propose a frequency planning methodology for LDACS A2A yielding no harmful interference towards the operation of DME and TACAN. This methodology can also be applied to the frequency planning of the LDACS A/G reverse link. Using a very conservative compatibility criteria, we apply the proposed methodology to assess the feasibility of the LDACS A2A frequency planning in the $960-1164 \mathrm{MHz}$ aeronautical L-band. We restrict our frequency planning to the north-east coast of North America, the North Atlantic Corridor, and western Europe.

The obtained results show that LDACS A2A will be able to operate in numerous frequency channels within the 960-1164 $\mathrm{MHz}$ frequency band without affecting the proper operation of DME and TACAN. In the North Atlantic Corridor, as soon as the LDACS A2A station is sufficiently separated from the mainland, the entire aeronautical L-band can be employed

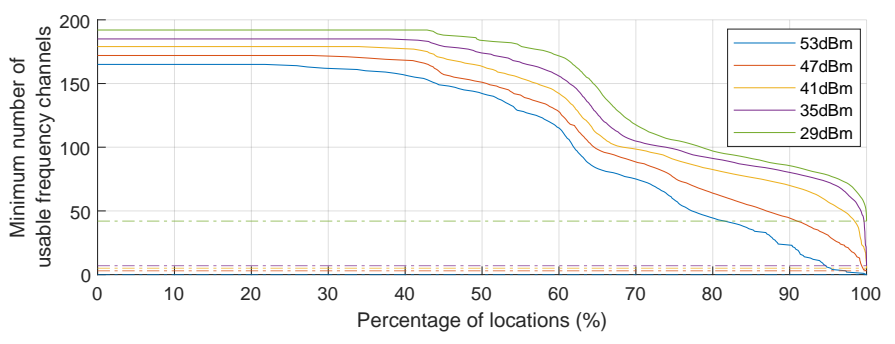

(a) Considering the frequency bands $960-1010 \mathrm{MHz}, 1050-1070$ $\mathrm{MHz}$, and $1110-f_{\max } \mathrm{MHz}$, where $f_{\max }$ is the maximum frequency channel allowed by the ITU Resolution 417 for each considered EIRP.

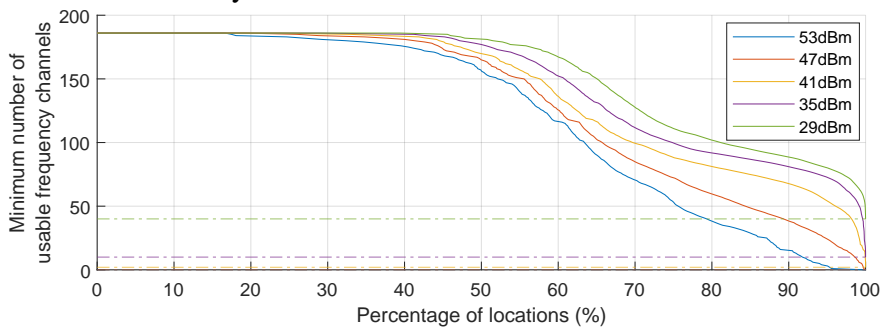

(b) Considering the LDACS A/G frequency bands: $964-1010 \mathrm{MHz}$ and $1110-1156 \mathrm{MHz}$.

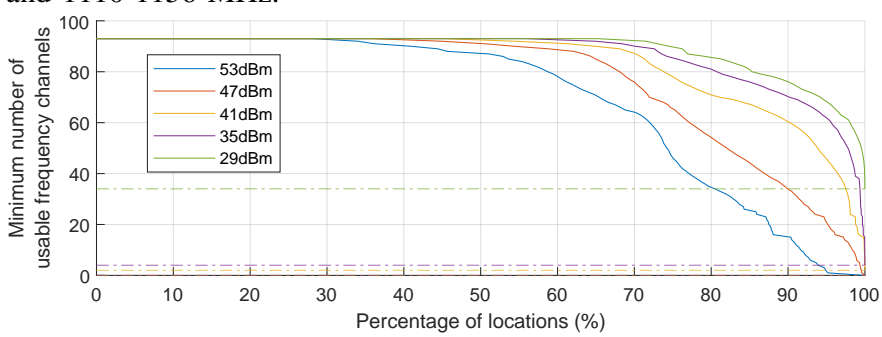

(c) Considering the LDACS A/G reverse link frequency band: 964$1010 \mathrm{MHz}$.

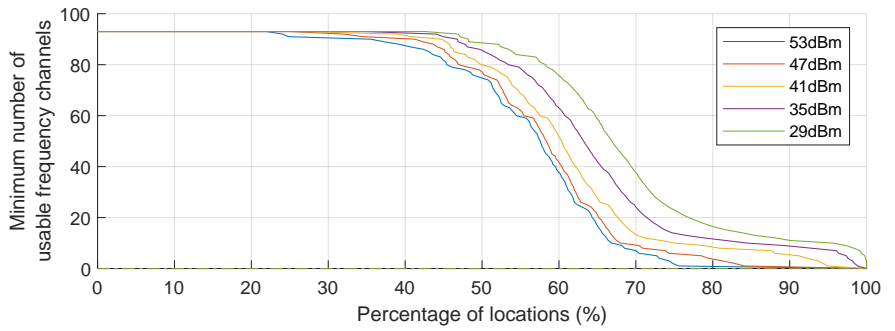

(d) Considering the LDACS A/G forward link frequency band: 1110$1156 \mathrm{MHz}$.

Fig. 11: Minimum number of usable frequency channels for a percentage of the locations and for different LDACS A2A EIRPs. Note the horizontal lines indicating the minimum number of frequency channels usable in any considered location for the different EIRPs. 
without disturbing the DME/TACAN operation. In the continental airspace, the number of usable frequency channels decreases but still remains high in most locations, especially in Europe. Although some small regions in North America present challenging compatibility conditions, our results show that LDACS A2A can operate anywhere in the region of interest, as multiple frequency channels are always usable. Even considering the compatibility with the systems operating at $1030 \mathrm{MHz}$ and $1090 \mathrm{MHz}$, as well as the ITU Resolution 417 safeguarding the RNSS receivers, LDACS A2A can still operate anywhere in the region of interest and numerous frequency channels can be used in most locations. Moreover, we see that LDACS A2A could benefit from adapting its EIRP dynamically. It could reduce the EIRP in the most challenging regions to be able to use more frequency channels, and increase it in the less challenging regions to, for example, increase its data throughput or communications range. In any case, the results clearly show that the lower part of the Lband, i.e., 960-1040 MHz, is the most promising frequency band for LDACS A2A operation, especially in the continental airspace. Thus, LDACS A2A could successfully operate in the LDACS A/G reverse link band, i.e., 964-1010 MHz. In addition, the frequencies $960-964 \mathrm{MHz}$ yield practically no interference towards DME/TACAN, and LDACS A2A would greatly benefit from operating there as well. In fact, some of these frequencies can be used anywhere in the considered region of interest, which might allow LDACS A2A to have a globally available frequency channel for its operation.

\section{ACKNOWLEDGMENT}

This work was co-funded under the research program LuFo V-3 ("Luftfahrtforschungsprogramm") of the German Federal Ministry of Economy and Energy (BMWi).

\section{REFERENCES}

[1] SESAR homepage: http://www.sesarju.eu/.

[2] NextGen homepage: https://www.faa.gov/nextgen/.

[3] M. A. Bellido-Manganell, "Design Approach of a Future Air-to-Air Data Link," in 2018 IEEE/AIAA 37th Digital Avionics Systems Conference (DASC), London, UK, Sep. 2018.

[4] T. Graupl, C. Rihacek, B. Haindl, and Q. Parrod, "LDACS A/G Specification," SESAR2020, Tech. Rep. PJ14-02-01 D3.3.030, Aug. 2019. [Online]. Available: https://www.ldacs.com/

[5] M. A. Bellido-Manganell, T. Gräupl, O. Heirich, N. Mäurer, A. FilipDhaubhadel, D. M. Mielke, L. M. Schalk, D. Becker, N. Schneckenburger, and M. Schnell, "LDACS Flight Trials: Demonstration and Performance Analysis of the Future Aeronautical Communications System," Submitted to the IEEE Transactions on Aerospace and Electronic Systems to be considered for publication, 2020.

[6] "Finalization of LDACS Draft SARPs," International Civil Aviation Organization, Second Meeting of DCIWG (Data Communication Infrastructure Working Group), Montreal, Tech. Rep. Working Paper WP05 including Appendix, Oct. 2018.

[7] M. Mostafa, M. A. Bellido-Manganell, and T. Graupl, "Feasibility of Cell Planning for the L-Band Digital Aeronautical Communications System Under the Constraint of Secondary Spectrum Usage," IEEE Transactions on Vehicular Technology, vol. 67, no. 10, pp. 9721-9733, Oct. 2018.

[8] Final Acts WRC-07. International Telecommunication Union, 2007.

[9] M. A. Bellido-Manganell, T. Graupl, and M. Schnell, "Impact Assessment of the L-Band Digital Aeronautical Communications System on the Joint Tactical Information Distribution System," IEEE Transactions on Vehicular Technology, vol. 68, no. 4, pp. 3629-3641, Apr. 2019.
[10] Final Acts WRC-15. International Telecommunication Union, 2015.

[11] U. Epple and M. Schnell, "Advanced Blanking Nonlinearity for Mitigating Impulsive Interference in OFDM Systems," IEEE Transactions on Vehicular Technology, vol. 66, no. 1, pp. 146-158, Jan. 2017.

[12] "Aeronautical Study - NAVAID Modernization," NAV CANADA, Tech. Rep., Aug. 2017.

[13] What is flying? database. Accessed March 3, 2021. [Online]. Available: https://whatisflying.com/

[14] "LDACS (L-band Digital Aeronautical Communications System) versus DME/TACAN compatibility," International Civil Aviation Organization, Navigation Systems Panel (NSP), Sixth Meeting of the Joint Working Groups, Virtual Meeting, Tech. Rep. Working Paper WP28 including Appendix, Jun. 2020. 